\title{
Front Matter: Volume 10224
}

, "Front Matter: Volume 10224," Proc. SPIE 10224, International Conference on Micro- and Nano-Electronics 2016, 1022401 (24 January 2017); doi:

10.1117/12.2270028

SPIE Event: The International Conference on Micro- and Nano-Electronics 2016, SPIE. 2016, Zvenigorod, Russian Federation 


\section{PROCEEDINGS OF SPIE}

\section{International Conference on Micro- and Nano-Electronics 2016}

Vladimir F. Lukichev Konstantin V. Rudenko Editors

\section{3-7 October 2016 \\ Zvenigorod, Russian Federation}

Organized by

Institute of Physics and Technology (Russian Federation)

Sponsored by

SC "Molecular Electronics Research Institute" (Russian Federation)

Russian Foundation for Basic Researches (Russian Federation)

Russian Academy of Sciences (Russian Federation)

Technolnfo Ltd. (United Kingdom/Russian Federation)

NIX Company (Russian Federation)

Published by

SPIE 
The papers in this volume were part of the technical conference cited on the cover and title page. Papers were selected and subject to review by the editors and conference program committee. Some conference presentations may not be available for publication. Additional papers and presentation recordings may be available online in the SPIE Digital Library at SPIEDigitallibrary.org.

The papers reflect the work and thoughts of the authors and are published herein as submitted. The publisher is not responsible for the validity of the information or for any outcomes resulting from reliance thereon.

Please use the following format to cite material from these proceedings:

Author(s), "Title of Paper," in International Conference on Micro- and Nano-Electronics 2016, edited by Vladimir F. Lukichev, Konstantin V. Rudenko, Proceedings of SPIE Vol. 10224 (SPIE, Bellingham, WA, 2016) Seven-digit article CID Number.

ISSN: 0277-786X

ISSN: 1996-756X (electronic)

ISBN: 9781510609495

ISBN: 9781510609501 (electronic)

Published by

SPIE

P.O. Box 10, Bellingham, Washington 98227-0010 USA

Telephone +1 3606763290 (Pacific Time) · Fax +1 3606471445

SPIE.org

Copyright @ 2016, Society of Photo-Optical Instrumentation Engineers.

Copying of material in this book for internal or personal use, or for the internal or personal use of specific clients, beyond the fair use provisions granted by the U.S. Copyright Law is authorized by SPIE subject to payment of copying fees. The Transactional Reporting Service base fee for this volume is $\$ 18.00$ per article (or portion thereof), which should be paid directly to the Copyright Clearance Center (CCC), 222 Rosewood Drive, Danvers, MA 01923. Payment may also be made electronically through CCC Online at copyright.com. Other copying for republication, resale, advertising or promotion, or any form of systematic or multiple reproduction of any material in this book is prohibited except with permission in writing from the publisher. The CCC fee code is 0277-786X/16/\$18.00.

Printed in the United States of America.

Publication of record for individual papers is online in the SPIE Digital Library.

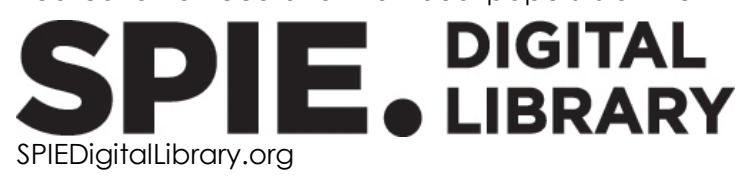

Paper Numbering: Proceedings of SPIE follow an e-First publication model. A unique citation identifier (CID) number is assigned to each article at the time of publication. Utilization of CIDs allows articles to be fully citable as soon as they are published online, and connects the same identifier to all online and print versions of the publication. SPIE uses a seven-digit CID article numbering system structured as follows:

- The first five digits correspond to the SPIE volume number.

- The last two digits indicate publication order within the volume using a Base 36 numbering system employing both numerals and letters. These two-number sets start with 00, 01, 02, 03, 04, 05, 06, 07, 08, 09, OA, OB ... 0Z, followed by 10-1Z, 20-2Z, etc. The CID Number appears on each page of the manuscript. 


\title{
Contents
}

\author{
xi Authors \\ xv Conference Committee \\ xxi Introduction
}

MICRO- AND NANOELECTRONIC MATERIALS AND FILMS I

$1022402 \quad \mathrm{HfO}_{2} / \mathrm{Pr}_{2} \mathrm{O}_{3}$ gate dielectric stacks [10224-45]

1022403 Low resistance Ti/Si/Ti/Al/Ni/Au ohmic contact for AIGaN/GaN HEMT [10224-66]

1022404 Tungsten alloyed with rhenium as an advanced material for heat-resistant silicon ICs interconnects [10224-10]

1022405 Metal-assisted chemical etching of silicon with different metal films and clusters: a review [10224-54]

1022406 Mechanical properties of bimetallic one-dimensional structures [10224-94]

1022407 Optical coefficients of nanometer-thick copper and gold films in microwave frequency range [10224-26]

1022408 Effective optical constants of silver nanofilms calculated in wide frequency range [10224-77]

1022409 Analysis of contribution from various order diffraction maxima to complex magnetooptical Kerr effect from three-dimensional structures like magnetophotonic crystals [10224-16]

10224 OA Modulation of magnetic interaction in Bismuth ferrite through strain and spin cycloid engineering [10224-68]

10224 OB Quantum-mechanical relaxation model for characterization of fine particles magnetic dynamics in an external magnetic field [10224-106]

MICRO- AND NANOELECTRONIC MATERIALS AND FILMS II

10224 OC GeSi nanocrystals formed by high temperature annealing of $\mathrm{GeO} / \mathrm{SiO}_{2}$ multilayers: structure and optical properties [10224-17]

10224 OD Hydrogenated amorphous silicon based p-i-n structures with Si and Ge nanocrystals in ilayers [10224-22] 
$10224 \mathrm{OE} \quad$ Investigation of the phase formation from nickel coated nanostructured silicon [10224-8]

$10224 \mathrm{OF} \quad$ Chemical surface treatment of $\mathrm{Ge}_{2} \mathrm{Sb}_{2} \mathrm{Te}_{5}$ thin films for phase change memory application [10224-103]

10224 OG Some important aspects in the glass structure of chalcogenide systems [10224-47]

$10224 \mathrm{OH} \quad$ Study of morphological characteristic of por-Si formed using metal-assisted chemical etching by BET-method and fractal geometry [10224-101]

10224 Ol Formation of nanoporous structure in silicon substrate using two-stage annealing process [10224-70]

$10224 \mathrm{~J} \quad$ Application of porous alumina formed in selenic acid solution for nanostructures investigation via Raman spectroscopy [10224-102]

10224 OK Low-threshold field emission in planar cathodes with nanocarbon materials [10224-92]

$10224 \mathrm{OL}$ The features of CNT growth on catalyst-content amorphous alloy layer by CVD-method [10224-65]

\section{PHYSICS OF MICRO- AND NANODEVICES}

10224 OM Low-dimensional transit-time diodes for terahertz generation [10224-1 10]

10224 ON Efficiency of the signal detection in RF and sub THz ranges by means of GaAs tunnel diodes [10224-73]

1022400 Electrically stimulated high-frequency replicas of a resonant current in GaAs/AIAs resonant-tunneling double-barrier THz nanostructures [10224-55]

10224 OP Photoresponse in graphene field effect transistor under ultra-short pulsed laser irradiation [10224-24]

$102240 Q \quad$ Dielectric influence on IV curve of graphene field effect transistor [10224-18]

10224 OR Sn nanothreads in GaAs: experiment and simulation [10224-109]

10224 OS Thin film ruthenium microstructures for transition edge sensors [10224-19]

10224 OT Investigation of memristor effect on the titanium nanowires fabricated by focused ion beam [10224-78]

10224 OU Resistive switching of vertically aligned carbon nanotube by a compressive strain [10224-40] 
10224 OV Simulation of field-effect transistors and resonant tunneling diodes based on graphene [10224-14]

10224 OW Ensemble Monte Carlo simulation of electron transport in GaAs/AIAs quantum wire structure under the effect of terahertz electric field [10224-43]

10224 OX Thermo injecting electrical instability in the AlxGa1-xAs/GaAs heterostructures with tunnelnontransparent potential barriers [10224-64]

10224 OY I-V characteristics simulation of silicon carbide Ti/4H-SiC Schottky diode [10224-80]

$102240 Z$ Development of drift-diffusion numerical models of high-speed on-chip photodetectors with heterojunctions [10224-33]

$1022410 \quad$ Numerical modeling of microwave switchers with subpicosecond time delay [10224-29]

1022411 Ambipolar memristor-based oscillator [10224-57]

1022412 The spatially dispersive eigenvalues of permittivity operator and frequency-dependent surface impedance for conductors without the dc dissipation [10224-79]

1022413 Calculation of the high-frequency conductivity and the Hall constant of a thin semiconductor film [10224-13]

1022414 A simple calculation method for heavy ion induced soft error rate in space environment [10224-99]

1022415 Compact modeling of radiation-induced drain leakage current [10224-104]

1022416 Total ionizing dose effects modeling in common-gate tri-gate FinfETs using Verilog-A [10224-56]

1022417 Application of triple modular redundancy for soft error mitigation in 65-28 nm CMOS VLSI [10224-98]

1022418 Layout-aware simulation of soft errors in sub-100 nm integrated circuits [10224-58]

\section{MICRO- AND NANOELECTROMECHANICAL SYSTEMS}

1022419 Silicon ohmic lateral-contact MEMS switch for RF applications [10224-82]

$102241 \mathrm{~A}$ A low actuation voltage bistable MEMS switch: design, fabrication and preliminary testing [10224-23]

10224 1B Research of the micromechanical three-axis accelerometer [10224-42]

10224 1C Simulation of heavy charged particles damage on MEMS [10224-38] 
10224 ID Anchored multi-DOF MEMS gyroscope having robust drive mode [10224-89]

$10224 \mathrm{IE} \quad$ Highly sensitive devices for primary signal processing of the micromechanical capacitive transducers [10224-31]

$10224 \mathrm{IF}$ Integral planar supercapacitor with CNT-based composite electrodes for heat-sensitive MEMS and NEMS [10224-75]

10224 IG Biosensor platform based on carbon nanotubes covalently modified with aptamers [10224-71]

$102241 \mathrm{H} \quad$ Angular MET sensor for precise azimuth determination [10224-72]

1022411 The simulation model of planar electrochemical transducer [10224-76]

$102241 \mathrm{~J}$ The planar silicon-based microelectronic technology for electrochemical transducers [10224-84]

$102241 \mathrm{~K}$ The precision seismometer based on planar electrochemical transducer [10224-85]

10224 IL 3D simulation of silicon micro-ring resonator with Comsol [10224-44]

MICRO- AND NANOELECTRONIC TECHNOLOGIES I

10224 IM Electro-optical converter of zero-order and second-order Bessel laser beams for the photolithography systems [10224-9]

$102241 \mathrm{~N}$ The formation of photoresist film with thicknesses from $\mathbf{0 . 7}$ microns to 100 microns on surfaces with considerable relief by spray coating on the heated substrate [10224-11]

1022410 Problems and prospects of maskless (B)EUV lithography [10224-91]

10224 IP Resistless lithography - selective etching of silicon with gallium doping regions [10224-20]

$102241 Q \quad$ Features of local anodic oxidation process [10224-6]

10224 IR Deposition of polymers on structures with nano-gaps fabricated between carbon nanotubes by focused ion beam etching [10224-37]

10224 is Direct laser patterning of graphene-based biosensors [10224-93]

\section{MICRO- AND NANOELECTRONIC TECHNOLOGIES II}

10224 IT III-Nitride advanced technologies and equipment for microelectronics [10224-100]

$102241 \mathrm{U}$ Plasma parameters and active species kinetics in $\mathrm{CF}_{4} / \mathrm{O}_{2} / \mathrm{Ar}$ gas mixture: effects of $\mathrm{CF}_{4} / \mathrm{O}_{2}$ and $\mathrm{O}_{2}$ /Ar mixing ratios [10224-21]

10224 IV Technology for fabrication of sub-20 $\mathbf{n m}$ silicon planar nanowires array [10224-87] 
$10224 \mathrm{IW}$ Comparative study of $\mathrm{CF}_{4}$ - and $\mathrm{CHF}_{3}$-based plasmas for dry etching applications [10224-35]

10224 IX Cellular-automata model of oxygen plasma impact on porous low-K dielectric [10224-32]

$102241 Y$ Investigation of the reactive ion etching of $\mathrm{Ge}_{2} \mathrm{Sb}_{2} \mathrm{Te}_{5}$ thin films [10224-97]

1022412 A study of the vertical walls and the surface roughness GaAs after the operation in the combined plasma etching [10224-90]

1022420 Low-damage plasma etching of porous low-k films in $\mathrm{CF}_{3} \mathrm{Br}$ and $\mathrm{CF}_{4}$ plasmas under lowtemperature conditions [10224-88]

$1022421 \quad$ Elements for hard X-ray optics produced by cryogenic plasma etching of silicon [10224-25]

1022422 Temperature switching waves in a silicon wafer on lamp-based heating [10224-30]

1022423 Critical parameters of silicon wafer lamp-based annealing in high power flux of incoherent radiation [10224-69]

1022424 Evolution of structural properties of Si(001) subsurface layer containing He bubbles by low temperature annealing [10224-62]

\section{METROLOGY AND DIAGNOSTICS}

1022425 Application of spectral ellipsometry to in situ diagnostics of atomic layer deposition of dielectrics on silicon and AIGaN [10224-34]

1022426 Non-destructive determination of thickness of the dielectric layers using EDX [10224-12]

1022427 TDR method for determine IC's parameters [10224-67]

1022428 Determination of mechanical stress in the silicon nitride films with a scanning electron microscope [10224-1]

1022429 Method of stress and measurement modes for research of thin dielectric films of MIS structures [10224-107]

10224 2A Numerical simulation of thin-film microthermocouple for the research of dissipation in tunneling contact [10224-36]

10224 2B Classification automation of thermoplastic particles in a cured epoxy matrix according to their size on microscopic images [10224-81]

10224 2C Fundamentals of the fast neutral beams diagnostics [10224-59]

10224 2D Optical emission 2D-tomography of plasma: case of rectangular two-view scanning and diagonal symmetry of inhomogeneities [10224-49] 
$102242 \mathrm{E}$ Experimental and theoretical investigations of quantum state transfer and decoherence processes in quasi-one-dimensional systems in multiple-quantum NMR experiments [10224-4]

$102242 F$ Quantum entanglement and quantum discord in dimers in multiple quantum NMR experiments [10224-5]

$102242 \mathrm{G}$ Multifunctional quantum node based on double quantum dot in laser and cavity fields [10224-51]

$102242 \mathrm{H} \quad$ Diamond chip under single-photon driving as a high spatial resolution quantum magnetometer and electrometer [10224-53]

$1022421 \quad$ Stark-shift based quantum dot-cavity electrometer [10224-96]

$102242 \mathrm{~J}$ Single-photon transmission and spectroscopy of diamond microring isomers [10224-48]

10224 2K Single-electron solitons in magnetic field [10224-111]

$10224 \mathrm{~L}$ Continuous-time quantum walk of two interacting fermions on a cycle graph [10224-83]

$102242 M \quad$ Quantum-classical crossover in quantum walks mixing time [10224-112]

$102242 \mathrm{~N}$ Broadband biphotons in the single spatial mode through high pump focusing and walk-off effect [10224-61]

1022420 Quantum states tomography with noisy measurement channels [10224-63]

$102242 \mathrm{P}$ Schmidt decomposition and multivariate statistical analysis [10224-60]

$102242 Q$ Study of higher order correlation functions and photon statistics using multiphotonsubtracted states and quadrature measurements [10224-52]

$102242 R \quad$ Analysis of quantum tomography protocol efficiency for triphoton polarization states [10224-46]

$102242 S \quad$ Numerical characteristics of quantum computer simulation [10224-74]

$102242 \mathrm{~T}$ Effective computation of quantum discord in a multiqubit spin chain [10224-86]

$102242 U$ Effect of crosstalk on QBER in QKD in urban telecommunication fiber lines [10224-39]

$102242 \mathrm{~V}$ Single photon detector design features [10224-50]

10224 2W Fast polarization QKD scheme based on $\mathrm{LiNbO}_{3}$ phase modulators [10224-28] 
$102242 X$ Computer simulation of quantum effects in Tavis-Cummings model and its applications [10224-108]

$102242 Y$ Dark states of atomic ensembles: properties and preparation [10224-7]

$102242 Z$ Selective measurement of quantronium qubit states by using of mesoscopic non-linear oscillator [10224-95] 
Proc. of SPIE Vol. 10224 1022401-10 Downloaded From: https://www.spiedigitallibrary.org/conference-proceedings-of-spie on 26 Apr 2023
Terms of Use: https://www.spiedigitallibrary.org/terms-of-use 


\section{Authors}

Numbers in the index correspond to the last two digits of the seven-digit citation identifier (CID) article numbering system used in Proceedings of SPIE. The first five digits reflect the volume number. Base 36 numbering is employed for the last two digits and indicates the order of articles within the volume. Numbers start with 00, 01, 02, 03, 04, 05, 06, 07, 08, 09, OA, OB...0Z, followed by 10-1Z, 20-2Z, etc.

Abdullaev, D. A., 1P, 26

Abramov, Igor I., OV Ageev, Oleg A., OT, OU, 1 Z

Aleksanyan, A. A., 00

Alekseyev, A., $1 \mathrm{~F}$

Alexeev, A. N., IT

Amirov, lldar I., $1 \mathrm{~A}$

Andreev, Dmitrii V., 29

Andreev, Valery G., 06, 07, 08

Andreev, Vladimir V., 29

Antonov, Alexander, $1 \mathrm{H}$

Avilov, V. I., OT

Avosopyants, G. V., 2Q, 2R

Baklanov, M., 20

Balbekov, A., 18

Bantysh, B. I., 20

Belinsky, L. V., 2Q, 2R

Belov, A. N., 04, $1 Q$

Berezkina, Alexandra YU., OE

Blinov, Yuriy F., OU

Bobkov, S., 18

Bobrinetskiy, Ivan I., OP, 1G, 1R, 1S

Bochkin, G. A., 2E

Bogdanov, YU. I., 2O, 2P, 2Q, 2R

Bogdanova, N. A., 2O, 2P, 2Q

Bondarev, F., 1B

Borshchevskaya, N. A., 2R

Borzdov, Andrei $\mathrm{V}$., OM, OW

Borzdov, Vladimir M., OM, OW

Boyko, Anton N., $\mathrm{OH}$

Bugaev, A., OR

Bulyarskii, S., OL

Chaly, V. P., IT

Chaplygin, YU. A., 04, OK, 1Q

Chekmachev, Vadim G., 21

Cherkov, A. G., OC

Chernyaev, A. P., 12

Chernyavskiy, A., 2S, 2T

Chesnokov, Yury M., 24

Chkhalo, N. I., 10

Chuev, M., OB

Ciesielski, R., OL

Clemente, Iosif E., 20, 25

Cohn, I. A., OS

Davydov, F., OM

Dedkova, A. A., 28

Degtyarev, S. A., $1 \mathrm{~L}$

Denisenko, M. V., $2 Z$

Denisenko, M., 1E
Denisenko, Yuri I., 01

Djuzhev, N. A., 28

Dolgov, A. N., $1 \mathrm{~J}$

Doronin, S. I., 2E

Dresvyannikov, M. A., ON, 12

Drosdetsky, M. G., 15

Dubkov, S., OL

Dudin, Alexander A., 05, OE

Duplinskiy, A., 2W

Dvurechendkii, A. V., OD

Efremov, A., 1U, IW

Egorchikov, A. E., 1J

Egorov, I. V., $1 \mathrm{~J}$

Elushov, I. V., 14

Emelianov, Aleksei V., OK, OP, IR

Eskov, Andrey V., $1 \mathrm{Z}$

Ezhova, O., 1B

Fadeev, A. V., 2D

Fajgar, R., OD

Fastovets, D. V., 2P

Fedichkin, Leonid E., OM, 2L, 2M

Fedorova, A. V., 2E

Fel'dman, E. B., 2E

Filippov, S., 2K

Fomchenkov, S. A., ID

Gaev, Dahir S., $\mathrm{OH}$

Galimov, A. M., 14

Gavrilin, I., IF

Gavrilov, Sergey A., 05, OE, OH, OJ, OL

Gerasev, S. A., 2F

Gergel, V. A., OX

Glukhenkaya, V. B., OF

Glushko, A., 1C

Golishnikov, A. A., 04

Golovin, A. V., 1G, 15

Gorbunov, Maxim S., 16, 18

Gornev, E. S., 11, $1 \mathrm{~J}$

Gornev, Evgeny S., $1 \mathrm{X}$

Gorshkova, N. M., OX

Gromov, D., OJ, OL, IF, IY

Gusev, E. E., 28

Gutshin, Oleg P., 1X

Ilin, A. S., OS

Ilin, Oleg I., OU

llina, Marina $\mathrm{V} ., \mathrm{OU}$

Isaeva, A., 1E

Jityaev, I. L., OT

Kalmykov, Rustam M., $\mathrm{OH}$

Kalnov, V., 19 
Kalugin, Viktor V., OG

Kamaev, G. N., OD

Kanapin, Alan A., 2U, 2W

Kargin, Nikolay I., 03, 0Q

Karuzskii, A. L., ON, 0O, 12

Katamadze, K. G., 2N, 2Q, 2R

Kateev, Igor $\mathrm{YU}$., 2H, 2J

Kazakov, I. P., 00

Kelm, E. A., 26

Khabibullin, R. A., OR, OX

Khabutdinov, R., OM

Khamitov, K., $2 S$

Khlybov, A., 27

Khonina, S. N., 1D, 1L, 1M

Khorin, I., 07

Kireev, Dmitry M., OP

Kiselev, Dmitrii A., 24

Kitsyuk, E., OL, IF

Klimin, Viktor S., OU, 1 Z

Kochubei, S. A., OD

Kolomejtseva, Natali $V_{\text {., OV }}$

Kolomiytsev, A. S., OT

Komarov, I. A., 1G, is

Konoplev, B., 10, 1B, 1E

Korobova, Natalia E., OG

Koroleva, Olga M., IA

Kostyukov, D. A., 04

Kovalenko, A. G., OS

Kozmin, A., $1 Y$

Kozyukhin, S. A., OF

Krasovitsky, D. M., IT

Kravchenko, Vladislav V., 2A

Krayushkina, E., OY

Krishtop, T. V., 11

Krishtop, Vladimir, 1H, 1I, 1J, 1K

Krivyakin, G. K., OD

Kudrya, V., 2C

Kulik, S. P., 2N, 2Q

Kulyamina, Darya A., 03

Kurenya, Alexey L., 22, 23

Kurochkin, Vladimir L., 2U, $2 \mathrm{~V}$

Kurochkin, Yuriy V., 2U, 2V, 2W

Kuznetsov, Yu. A., 2R

Kuznetsova, E. I., 2F

Kuznetsova, I. A., 13

Kvasnyy, A. B., 20

Kwon, Kwang-Ho, IU, IW

Labunov, Vladimir A., OV

Ladunov, Vitalii $Y ., 2 X$

Lazarenko, P. I., OF, IY

Lebedev, E., IF

Lebedev, K., 19

Lee, Junmyung, $1 \mathrm{U}$

Lemeshko, S., $1 Q$

Levin, Denis D., IN

Lomov, Andrey A., 24

Lukichev, Vladimir F., 19, 21, 2O, 2P, 2Q

Lysenko, I., 1B, 1E

Maishev, Yu., 2C
Makarchuk, V., IC

Malakhanov, A., OY

Maltsev, P. P., OR, OX

Mamaev, V. V., IT

Maniecki, T., OL

Maslovsky, Vladimir M., 29

Matyushkin, Igor $V_{\text {., }} 1 \mathrm{X}$

Melnikov, Alexey A., 2L

Meshchaninov, Fedor, 2M

Miakonkikh, Andrey V., 19, 1V, 20, 21, 24, 25

Mierczynski, P., OL

Mikhailova, M. S., OF

Mikhaylichenko, S., 1C

Miller, Alexander V., $2 U$

Milovanov, R. A., 1P, 26

Minaev, Viktor S., OG

Minkin, V. S., OX

Mischenko, I., OB

Mityagin, Yu. A., 0O, 12

Molchanova, A., 02

Morgunov, A., IW

Murzin, V. N., 00

Naumov, Victor $V_{\text {., }} 1 \mathrm{~A}$

Nazarkina, Y. V., OJ

Nemtseva, S. Y., OF

Nevolin, Vladimir K., IN

Nikitov, S. A., OX

Novikov, A. V., $1 \mathrm{~J}$

Orlikovsky, N., 07

Orlov, V. V., 15

Otero, Nerea, OP

Otsuji, T., OR

Ovcharov, Vladimir V., 22, 23

Ozhigov, Yuri I., 2X, $2 Y$

Panchenko, P., OY

Paporkov, Vladimir A., 09

Paranin, V. D., $1 M$

Patiukov, N., 28

Pavelyev, V.S., 1D

Pavlov, A. Yu., OX

Pavlov, A., 05, OK, OL

Pavlovskiy, V. V., OX

Perestoronin, A. V., 0N, 0O, 12

Petrov, S. I., IT

Petukhov, V., OK

Petukhov, Vladimir A., 2A

Pillai, Shreeja, OA

Pisarenko, I. V., $0 Z$

Podlipnov, V. V., $1 \mathrm{~L}$

Polkovnikov, V. N., 10

Polohin, A. A., OJ

Ponomarev, D., OR

Popov, V. G., 1J

Prigara, Valeriya P., 22, 23

Priimochenko, V. V., ON

Prokaznikov, Alexander V., 09

Pronin, S., 07

Purkrt, A., OD 
Putrya, M. G., 04

Pyatilova, Olga $\vee ., 05,0 \mathrm{E}, \mathrm{OH}$

Radkov, A., OY

Rakitin, Alexander V., 11

Rakitin, Vladimir V., 11

Rana, D. S., OA

Remes, Z., OD

Reshi, Hilal Ahmad, OA

Rezvanov, Askar, $1 \mathrm{X}$

Rinnert, H., OC

Rodionov, D., 27

Rogachev, Maxim S., 2J

Rogozhin, Alexander E., 02, 07, 19, 1V, 21

Romanov, D. N., 13

Romanova, Irina A., OV

Romashkin, Alexey V., 1N, 1R

Romero, Pablo, OP

Rozanov, Roman Yu., IN

Rubtsova, E. I., 1G, 15

Rudakov, Valery I., OI, 22, 23

Rudenko, Konstantin V., 0M, 19, 1V, 20, 21, 2D

Rudenko, M., 2K

Ryazanov, R., $1 \mathrm{~F}$

Rybalka, S., OY

Ryndin, E. A., OZ, 10, 1B, $1 \mathrm{E}$

Ryzhii, M., OR

Ryzhii, V., OR

Ryzhuk, Roman V., 03

Sablina, Victoria A., 2B

Safonov, S. O., 04

Sagunova, I., 1Q

Salashchenko, N. N., 10

Saletsky, Alexander M., 06

Satanin, A. M., $2 Z$

Savenko, O. V., 13

Savitskiy, A., 05

Semenikhin, I., OM, OR

Shabadarova, D., IW

Shabalina, A. S., IK

Shakhnov, V., 1C

Shaman, Y.P., OJ

Shamanaev, A., OK

Shcherbachev, Kirill D., 24

Shelke, Vilas, OA

Sherchenkov, A. A., OF, IY

Shevyakov, V. I., 04, 1Q

Shilyaeva, Yulia I., OE

Shklyaev, A. A., OD

Shmelev, S. S., 00

Shostachenko, Stanislav A., 03, 0Q

Shuliatyev, A., 05, 1 Y

Sidorov, F., 02

Sidorov, L. N., 26

Sitnikov, Ivan I., 06

Skorobogatov, A. P., 17

Skovoroda, Nikita A., $2 X$

Smelova, Ekaterina M., 06

Smirnov, Dmitry I., OE

Smirnov, Vladimir A., OT, $1 Z$
Snigirev, Anatoly A., 21

Sokolov, Alexander S., $2 \mathrm{U}$

Sokolov, S. A., 26

Solodovnik, Maxim S., $1 Z$

Stanishevskiy, Yaroslav M., OQ

Stoffel, M., OC

Stolyarov, Alexander A., 29

Stuchlik, J., OD

Stuchliková, T. H., OD

Svintsov, Dmitry, OM, 2K

Sysa, Artem V., 05, OE, IF, IY

Tatarintsev, Andrey A., 07, 19, 1V

Teplov, A., $2 S$

Terekhov, V., 1C

Tereshonok, D. V., 11

Timoshenkov, Sergey P., OF, OG, OH

Timoshenkov, V., OK, 27

Tominov, Roman V., $1 Z$

Toropov, M. N., 10

Trifonov, A., OL

Trofimov, A. A., OX

Tsarik, K. A., IR

Tskhovrebov, A. M., ON, 00

Tsukanov, Alexander V., 2G, 2H, 2I, 2J

Tsysar, Kseniya M., 06, 08

Uma, B. V., 1D

Ustimchik, V., 2W

Uvarov, llya V., 1A

Vaganova, Elena I., IA

Varnavsky, Alexander N., 2B

Varnavsky, Andrei N., 2B

Vasil'ev, S. G., 2E

Vdovin, V.I., OC

Vdovin, Vladimir A., 06, 07, 08

Vergeles, S. S., 11

Vergnat, M., OC

Verhovtseva, A. V., OX

Verma, Payal, 1D, $1 \mathrm{~L}$

Vishnevskiy, A., 20

Voevodin, V., $2 S$

Voevodin, VI., $2 S$

Volchkov, N. A., 12

Volodin, V. A., OC, OD

Vystavkin, A. N., OS

Vyurkov, Vladimir, OM, OR, OW 2K

Yachmenev, A., OR

Yadav, Rama Shanker, OA

Yunkin, Vyacheslav A., 21

Zaitsev, Dmitry, $1 \mathrm{H}$

Zaitsev, Sergey V., 2V

Zakharchenko, Roman $V_{\text {., }} 03,0 Q$

Zarev, Ivan S., 09

Zebrev, Gennady I., OQ, 14, 15, 16

Zelensky, Vladimir S., 06

Zherikhina, L. N., ON

Zhevnenko, D. A., 11

Zhigalov, $\mathrm{V}$., OK

Zinchenko, L., IC 
Zubov, D., 1P

Zvezdin, Nickolay Yu., 09

xiv

Proc. of SPIE Vol. 10224 1022401-14

Downloaded From: https://www.spiedigitallibrary.org/conference-proceedings-of-spie on 26 Apr 2023 Terms of Use: https://www.spiedigitallibrary.org/terms-of-use 


\title{
Conference Committee
}

\author{
Conference Chairs
}

Evgeny P. Velikhov, Russian Scientific Center "Kurchatov Institute" (Russian Federation)

Yuri V. Gulyaev, Kotel'nikov Institute of Radio Engineering and Electronics (Russian Federation)

\section{Conference Co-Chair}

Gennadiy Ya. Krasnikov, SC "Molecular Electronics Research Institute" (Russian Federation)

International Advisory Committee

Alexander L. Aseev, Rzhanov Institute of Semiconductor Physics (Russian Federation)

Dmitri V. Averin, Stony Brook University (United States)

Mikhail R. Baklanov, International Microelectronic Center (Belgium)

Francis Balestra, Grenoble Institute of Technology (France)

Vladimir A. Labunov, Belarusian State University of Informatics and Radioelectronics (Belarus)

Konstantin K. Likharev, Stony Brook University (United States)

Alexey N. Nazarov, Institute of Semiconductor Physics (Ukraine)

Jun-ichi Nishizawa, Semiconductor Research Institute (Japan)

Konstantin S. Novoselov, University of Manchester (United Kingdom)

Taiichi Otsuji, Tohoku University (Japan)

Iwo W. Rangelow, University of Ilmenau (Germany)

Stefan E. Schulz, Fraunhofer Institute for Electronic Nanosystems (Germany)

Thomas Skotnicki, ST Microelectronics (France)

Robert A. Suris, loffe Institute (Russian Federation)

Akira Toriumi, University of Tokyo (Japan) 
Program Committee

Chair

Yuri V. Gulyaev, Kotel'nikov Institute of Radio Engineering and Electronics (Russian Federation)

Co-Chairs

Igor G. Neizvestnyi, Rzhanov Institute of Semiconductor Physics

(Russian Federation)

Vladimir F. Lukichev, Institute of Physics and Technology

(Russian Federation)

Members

Vladimir B. Betelin, Scientific Research Institute for System Analysis (Russian Federation)

Yuri I. Bogdanov, Institute of Physics and Technology (Russian Federation)

Yuri A. Chaplygin, National Research University of Electronic Technology (Russian Federation)

Mikhail A. Chuev, Institute of Physics and Technology (Russian Federation)

Leonid E. Fedichkin, Moscow Institute of Physics and Technology, State University (Russian Federation)

Fadey F. Komarov, Belarusian State University (Belarus)

Peter S. Kop'ev, loffe Institute (Russian Federation)

Peter P. Maltsev, Institute on Ultra High Frequency Semiconductor Electronics (Russian Federation)

Vladislav Ya. Panchenko, Institute on Laser and Informatics Technologies (Russian Federation)

Dmitry V. Roshchupkin, Institute of Microelectronics Technology and High Purity Materials (Russian Federation)

Konstantin V. Rudenko, Institute of Physics and Technology (Russian Federation)

Alexander S. Rudy, P.G. Demidov Yaroslavl State University (Russian Federation)

Nikolay N. Salaschenko, Institute for Physics of Microstructures (Russian Federation)

Kev M. Salikhov, Kazan E.K. Zavoisky Physical-Technical Institute (Russian Federation)

Alexander S. Sigov, Moscow Technological University (Russian Federation)

Pavel A. Todua, Moscow Institute of Physics and Technology, State University (Russian Federation)

Vladimir V. Vyurkov, Institute of Physics and Technology (Russian Federation) 
Organizing Committee

Chair

Vladimir F. Lukichev, Institute of Physics and Technology

(Russian Federation)

Co-Chair

Konstantin V. Rudenko, Institute of Physics and Technology

(Russian Federation)

Members

Igor I. Abramov, Belarusian State University of Informatics and Radioelectronics (Belarus)

Ildar I. Amirov, Institute of Physics and Technology, Yaroslavl Branch (Russian Federation)

Yuri I. Bogdanov, Institute of Physics and Technology

(Russian Federation)

Anastas A. Buharaev, Kazan E.K. Zavoisky Physical-Technical Institute (Russian Federation)

Mikhail A. Chuev, Institute of Physics and Technology (Russian Federation)

Alexander A. Gorbazevitch, P.N. Lebedev Physical Institute (Russian Federation)

Eugeny S. Gornev, SC "Molecular Electronics Research Institute" (Russian Federation)

Mikhail A. Korolev, National Research University of Electronic Technology (Russian Federation)

Oleg P. Pchelyakov, Rzhanov Institute of Semiconductor Physics (Russian Federation)

Vladimir P. Popov, Rzhanov Institute of Semiconductor Physics (Russian Federation)

Vladislav Yu. Rubaev, NIX Company (Russian Federation)

Local Organizing Committee

Vladimir F. Lukichev - Chair of the Local Organizing Committee

Konstantin V. Rudenko - Co-Chair of the Local Organizing Committee

Yuri I. Bogdanov - Chair of the Extended Session

"Quantum Informatics"

Vladimir P. Kudrya - Scientific Secretary

Sergey I. Skalkin - Financial Director

Alexander N. Astakhov - Administrator

Alexey M. Dianov - PR-support

Andrey Yu. Chernyavskiy - WEB design

Andrey V. Miakonkikh - Technical Support

Alexander E. Rogozhin - Technical Support

Igor A. Semenikhin - Visa support 
Vladimir V. Vyurkov - Contacts to the invited speakers

Lidiya M. Besschastnova - Registration and accommodation

Irina Yu. Lukianova - Registration and accommodation

Inna V. Nikitushkina - Conference fee manager

Irina B. Novojilova - Conference fee manager

Session Chairs

Plenary Session I

Vladimir F. Lukichev, Institute of Physics and Technology

(Russian Federation)

Plenary Session II. Quantum Informatics I

Yuri I. Bogdanov, Institute of Physics and Technology

(Russian Federation)

Plenary Session III

Vladimir F. Lukichev, Institute of Physics and Technology

(Russian Federation)

1 Materials and Films I

Andrey V. Miakonkikh, Institute of Physics and Technology

(Russian Federation)

2 Physics of Nanotransistors

Vladimir V. Vyurkov, Institute of Physics and Technology

(Russian Federation)

3 Quantum Informatics II

Sergey P. Kulik, Lomonosov Moscow State University

(Russian Federation)

$4 \quad$ Advanced Lithography

Konstantin V. Rudenko, Institute of Physics and Technology

(Russian Federation)

5 Physics of Memory Cells

Oleg S. Trushin, Institute of Physics and Technology, Yaroslavl Branch

(Russian Federation)

6 Quantum Informatics III

Sergei A. Moiseev, Kazan Quantum Center, Kazan Scientific Research Technical University (Russian Federation) 
$7 \quad$ Materials and Films II

Andrey V. Miakonkikh, Institute of Physics and Technology

(Russian Federation)

$8 \quad$ Superconducting Devices

Vladimir F. Lukichev, Institute of Physics and Technology

(Russian Federation)

9 Quantum Informatics IV

Eduard B. Fel'dman, Institute of Problems of Chemical Physics

(Russian Federation)

10 Superconducting and Spintronics Devices

Valery G. Andreev, Lomonosov Moscow State University

(Russian Federation)

11 Meeting of the International Association of the Academies of Sciences I

Vladimir F. Lukichev, Institute of Physics and Technology

(Russian Federation)

12 Quantum Informatics V

Farid M. Ablayev, Kazan Federal University (Russian Federation)

13 Plasma-Based Technologies

Konstantin V. Rudenko, Institute of Physics and Technology

(Russian Federation)

14 Semiconductor Devices of Photonics

Vladimir V. Vyurkov, Institute of Physics and Technology

(Russian Federation)

15 Quantum Informatics VI

Andrey Chernyavskiy, Institute of Physics and Technology

(Russian Federation)

16 Modeling and Simulation of Semiconductor Devices

Vladimir V. Vyurkov, Institute of Physics and Technology

(Russian Federation)

17 Metrology and Characterization

Andrey V. Miakonkikh, Institute of Physics and Technology (Russian Federation) 
18 Quantum Informatics VII

Yuri I. Ozhigov, Lomonosov Moscow State University

(Russian Federation)

19 MEMS and NEMS

Ildar I. Amirov, Institute of Physics and Technology, Yaroslavl Branch (Russian Federation)

20 Meeting of the International Association of the Academies of Sciences II

Vladimir F. Lukichev, Institute of Physics and Technology

(Russian Federation) 


\section{Introduction}

The volume contains selected papers presented at the International Conference "Micro- and Nanoelectronics - 2016" (ICMNE-2016) which has been held in Zvenigirod, Moscow Region, Russia during October 3-7, 2016. ICMNE is a biannual conference covering the main fields of micro- and nanoelectronic technologies and device physics. Since 1992 the Institute of Physics and Technology (Moscow, Russia) is the permanent organizer of ICMNE. From 2003 ICMNE is an SPIE-affiliated conference. ICMNE-2016 included the Extended Session "Quantum Informatics2016". The ICMNE-2016 scope contained such scientific and technological fields as micro- and nanoelectronic materials and films, technologies and equipment, metrology, physics and technologies of micro- and nanodevices, simulation and modeling, MEMS and NEMS physics and technology, quantum informatics. ICMNE-2016 included three plenary sessions and 18 topical sessions covering the following areas of focus:

- Physics of Nanotransistors, Photonic Devices, and Memory Cells

- Materials and Films

- Advanced Lithography

- Superconducting and Spintronics Devices

- Plasma-Based Technologies

- Quantum Informatics

- Simulation and Modeling and Simulation of Semiconductor Devices

- MEMS and NEMS

- Metrology and Characterization

The scientific program was based on invited and contributed papers from the scientists employed at European and Siberian Regions of Russia, Azerbaijan, Belarus, Poland, Germany, France, Korea, Japan, and India. The invited lectures on the current achievements and challenges in the contemporary microelectronics were delivered by the scientists from Germany, Japan, and Russia. The contributions to the sessions of the Conference were made by academic institutions, universities as well as from the industry. More than 100 contributions were discussed at oral presentations; about 120 others were presented as posters.

We hope that helpful discussions of these works at the sessions of the Conference and during personal contacts between attendees will promote the research activity in microelectronic community. Additional information about ICMNE-2016 can be found at the conference website http://www. icmne.ftian.ru

Vladimir F. Lukichev 
Proc. of SPIE Vol. 10224 1022401-22 Downloaded From: https://www.spiedigitallibrary.org/conference-proceedings-of-spie on 26 Apr 2023
Terms of Use: https://www.spiedigitallibrary.org/terms-of-use 